\title{
Human Body Orientation Estimation using a Committee based Approach
}

\author{
Manuela Ichim ${ }^{1}$, Robby T. Tan ${ }^{2}$, Nico van der $\mathrm{Aa}^{3}$ and Remco Veltkamp ${ }^{2}$ \\ ${ }^{1}$ University Politehnica of Bucharest, Bucharest, Romania \\ ${ }^{2}$ Utrecht University, Utrecht, The Netherlands \\ ${ }^{3}$ Noldus Information Technology, Wageningen, The Neterlands \\ ichim.manuela@gmail.com,r.t.tan@uu.nl,n.vanderaa@noldus.nl,r.c.veltkamp@uu.nl
}

\begin{abstract}
Keywords: Human Body Orientation, Neural Network, Support Vector Machine, Gaussian Mixture Models, PCA, Supervised Learning.

Abstract: $\quad$ Human body orientation estimation is useful for analyzing the activities of a single person or a group of people. Estimating body orientation can be subdivided in two tasks: human tracking and orientation estimation. In this paper, the second task of orientation estimation is accomplished by using HoG descriptors and other cues such as the velocity direction, the presence of face, and temporal smoothness. Three different classifiers: Gaussian Mixture Model, Neural Network and Support Vector Machine, are combined with the information from those cues to form a committee. The performance of the method is evaluated and the contribution to the final prediction of each classifier is assessed. Overall, the performance of the proposed approach outperforms the state-of-the-art method, both in terms of estimation accuracy, as well as computation time.
\end{abstract}

\section{INTRODUCTION}

The estimation of human body orientation is a task with potential use in many areas of modeling human activity and interaction. Determining how people move in an environment is a key step in understanding their actions. Among other applications, video surveillance systems can benefit from the task.

The goal of this paper is to estimate the body orientation of multiple human targets from a video sequence captured by a single view moving camera, as shown in Figure 1. Accomplishing this goal requires a few stages including human body detection and tracking. Additional computation, such as determining real-world 3D position coordinates of the targets and velocity orientation, can improve the results.

Estimating human body orientation can be formulated as a classification task with multiple classes of body rotation angles, where in this paper they are 8 distinct classes. The appearance of human targets is modeled by a dense grid of HoG descriptors (Dalal and Triggs, 2005), which are robust to scaling and light conditions, thus increasing the consistency of appearances within a given class. Additional cues are also used, such as the velocity orientation of the targets, the presence of face, temporal smoothing, etc.

A few methods have been introduced in the literature. Chen et al. (Chen and Odobez, 2012) as-

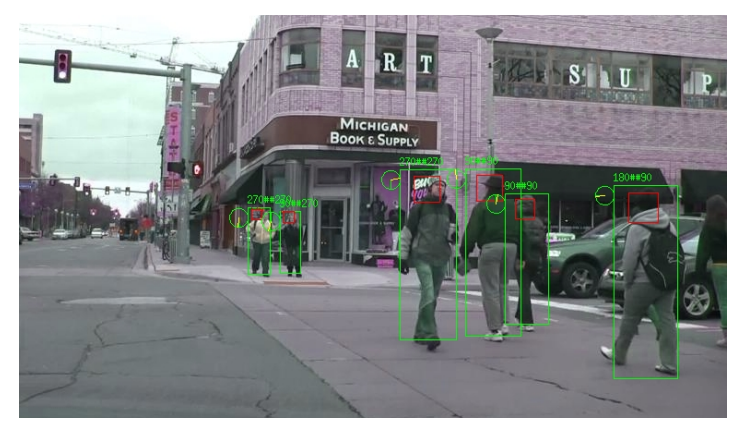

Figure 1: Output of our method. The green boxes are the detected humans. The red boxes are the detected heads. The first numbers are our estimated orientations, and the second numbers are the references.

sume that bounding boxes for the bodies and heads of the targets are given and information regarding their velocity direction and velocity magnitude are known. The final result of the algorithm consists of orientation estimations for head and body. They use the kernel based formulation to solve the problem. Tosato et al.(Tosato et al., 2012) address the problem of human orientation estimation by introducing a novel descriptor, Weighted ARay of COvariances (WARCO). This descriptor is based on the covariance of the features, which has been previously used for pedestrian detection. WARCO enables the classification of human targets possible despite some noisy pixels.

Lu et al. (Lu and Little, 2006) consider a template- 
based framework for tracking and recognizing athletes' actions using only visual information. The considered targets are encoded with a PCA-HoG descriptor, obtained by applying Principal Component Analysis (PCA) to the Histogram of Oriented Gradients (HoG) descriptor. This ensures a robust representation under variations in illumination and scale, while keeping computational costs low.

In this paper, we offer four main contributions: First, we introduce a method that incorporates a set of different classifiers and cues, allowing us to be more flexible in choosing the classification methods, and to have the best results obtained from the combined response from several classifiers (committee). Second, the way velocity information is taken into consideration. In the existing method (Chen and Odobez, 2012), the classes corresponding to the velocity angle class and adjacent ones are favored over the others, provided the magnitude of the velocity was above a certain threshold. Because of the greater flexibility of our method, we can model the velocity as a pseudo-classifier using a Gaussian distribution centered around the class indicated by the velocity direction. Third, the use of face detection. An important cue which allows human individuals to recognize and estimate the orientation of other human targets is the presence of the face. Face detection can be made relatively fast and is reliable, provided a minimal set of image quality are met. Fourth, the utilization of temporal information. The state of the art method (Chen and Odobez, 2012) considers the features of the targets independently from one frame to the other. However, since the video frames represent successive moments in time, and since human targets cannot abruptly change their orientation in a short amount of time (as between two consecutive frames), it is also reasonable to include temporal information in the estimation process.

\section{PROBLEM DESCRIPTION}

The input data of the system is a video sequence from a single view moving camera, depicting one or more human targets moving freely into, within and away from the scene. The goal is to estimate the orientation angle around the vertical axis of the body for each human target at each frame of the video. The output values of the angle are discretized into 8 distinct classes: $\{0,45,90,135,180,225,270,315\}$ degrees or, alternatively, $\{\mathrm{E}, \mathrm{NE}, \mathrm{N}, \mathrm{NW}, \mathrm{W}, \mathrm{SW}, \mathrm{S}, \mathrm{SE}\}$, where $\mathrm{S}$ or 270 degree represents the front direction, and $\mathrm{N}$ or 90 degree represents the back direction, as shown in Figure 2.

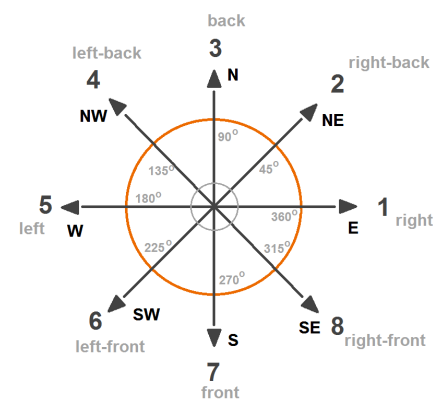

Figure 2: The 8 classes of the body orientations.

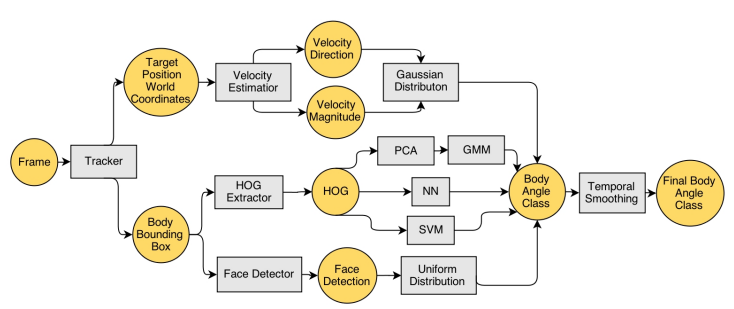

Figure 3: Pipeline of our body orientation estimation.

\section{COMMITTEE BASED CLASSIFICATION METHOD}

\subsection{Pipeline}

The pipeline for estimating the body orientation using our proposed method is summarized in the diagram in Figure 3. The input is video frames where human tracking is performed using the method described in (Choi et al., 2012), which is preferred over other tracking methods as the input is allowed to be originated from a single moving camera. Besides, the method is able to provide the estimates of the positions of human targets in the real world coordinate system. This information is particularly useful to determine the velocity direction and magnitude of the targets, which is an important cue at a later stage.

Aside from the coordinates of the targets, the method also returns bounding boxes of the targets. From these bounding boxes HoG descriptors (Dalal and Triggs, 2005) are extracted. These are then supplied to several pre-trained classifiers (PCA+GMM, Neural Network and Support Vector Machine), which produce probability estimates for each of the 8 angle classes.

Face is an important cue, since it restricts the plausible angle values. To include this information, face detection is performed on the bounding boxes. To maintain the consistency of the probabilistic framework, a uniform distribution based on the presence or absence of a face is generated. 
Another information is velocity direction and magnitude. This information can be integrated in the framework by fitting a standard Gaussian distribution centered around the velocity direction of an angle class, in such a way that a relatively high velocity yields a high probability for the frontal direction, and low for the other directions; while, a relatively low velocity yields the same probability for all directions.

The response from all the above classifiers and additional cues are combined and the estimated angle is considered to be the one with the highest probability. However, the final result is filtered using a sliding window. This additional step is performed to ensure the temporal smoothness of the change in orientation and to minimize the effect of misclassifications.

\subsection{Probabilistic Framework}

The core idea of HoG based classification using multiple classifiers is based on a probabilistic framework, where the task of estimating the orientation of a particular target at a given moment in time (frame) can be expressed as:

$$
\alpha^{*}=\arg \max _{\alpha} P(\alpha \mid \mathbf{x})
$$

where $\alpha$ represents the desired angle class, having 8 possible values. $\mathbf{x}=\left(\mathbf{x}^{b}, \mathbf{x}^{h}, v_{d}, v_{m}, f_{d}\right)$ variable encompasses the information known about the target, namely its HoG features for the body $\left(\mathbf{x}^{b} \in \mathbb{R}^{2268}\right)$, velocity direction $v_{d} \in\{1,2, \ldots, 8\}$, velocity magnitude $v_{m} \in \mathbb{R}$ and face detection $f_{d} \in\{0,1,2,3\}$ (0 meaning no face detection, 1 meaning left facing face detection, 2 meaning frontal face detection and 3 meaning right facing face detection).

Maximizing Eq.(1) is proportional to maximize the likelihood $P(\mathbf{x} \mid \alpha)$, which is determined by the combined response of the classifiers and cues mentioned in the pipeline, and can be expressed as:

$$
\begin{aligned}
P(\mathbf{x} \mid \alpha) & \propto \exp \left\{l_{G M M}(\mathbf{x} \mid \alpha)+l_{N N}(\mathbf{x} \mid \alpha)+l_{S V M}(\mathbf{x} \mid \alpha)\right. \\
& \left.+l_{\text {velocity }}(\mathbf{x} \mid \alpha)+l_{\text {face }}(\mathbf{x} \mid \alpha)\right\}
\end{aligned}
$$

where $l_{G M M}(\mathbf{x} \mid \alpha), l_{N N}(\mathbf{x} \mid \alpha), l_{S V M}(\mathbf{x} \mid \alpha), l_{\text {velocity }}(\mathbf{x} \mid \alpha)$ and $l_{\text {face }}(\mathbf{x} \mid \alpha)$ denote the log-likelihood given by the Gaussian Mixture Model classifier, Neural Network classifier, Support Vector Machine classifier, velocity cue and face detection cue, respectively. Details on the definitions of each of these likelihoods are given in the following subsections.

Our decision of using a combination of classifiers, rather than a single one is based on the argumentation given in (Bishop, 2007) which points out that the overal error of the committee can only improve the average error of each individual classifier. Due to the variability in response of each classifier, the overall error is expected to be better.

\subsection{Gaussian Mixture Model}

For each of the 8 classes, a Gaussian mixture model is computed based on the data points in the training dataset belonging to that class. Thus, the likelihood associated with the Gaussian Mixture Model (GMM) classifier is:

$$
\begin{aligned}
l_{G M M}(\mathbf{x} \mid \alpha) & =\log P_{G M M}(\mathbf{x} \mid \alpha) \\
& =\log \sum_{j=1}^{C} \pi_{j}^{(\alpha)} \mathcal{N}\left(\mathbf{x}^{b} \mid \mu_{j}^{(\alpha)}, \Sigma_{j}^{(\alpha)}\right)
\end{aligned}
$$

where $C$ represents the number of components in a Gaussian Mixture, $\mathcal{N}$ denotes the Gaussian distribution, $\pi_{j}^{(\alpha)}$ is a weighting factor and $\mu_{j}^{(\alpha)}$ and $\Sigma_{j}^{(\alpha)}$ represent the mean and covariance of each Gaussian distribution. Note that the subscript indicates the Gaussian within the Gaussian Mixture of a class, while the superscript indicates the angle class. Fitting each Gaussian Mixture onto the training data points of a given class is accomplished using the standard ExpectationMaximization (EM) algorithm (Bishop, 2007).

\subsection{PCA}

One of the limitations of the GMM is that the maximum likelihood estimation tends to produce singular or near-singular covariance matrices if the data occupies high-dimensional though actually lies on a lower dimensional manifold (which is usually the case in practice (Wang, 2011)). This happens as a Gaussian distribution, part of the mixture, is driven towards modeling a single data point. Another limitation of the GMM is that fitting over high-dimensional data is a slow process, since the EM training algorithm is iterative and at each iteration covariance matrices are computed for each Gaussian distribution in the mixture.

To mitigate the above mentioned limitations, we reduce the dimensionality of the HoG descriptors before using the GMM model for classification. One effective method is Principal Component Analysis. PCA can be defined as the orthogonal projection of the data onto a lower dimensional linear space, such that the variance of the projected data is maximized (Hotelling, 1933). Because the variance of the data is maximized, the separation between the points belonging to different classes is preserved as much as possible. Additionally, the PCA can discard the redundant 
and noisy information, thus improve the classification process.

\subsection{Neural Network}

The second HoG based classifier to be included in the committee is a Neural Network (NN). This method handles the high dimensionality of the data in a more natural way compared to PCA. The feed forward neural network can be regarded as an approach to fix the number of basis functions (represented here by the individual neurons), but to allow them to be adaptive (represented by the connection weights between the neurons, which can be regarded as parameters adapted during training). Furthermore, the extraction of relevant features in the data and the classification process are merged together. The disadvantage of this flexibility in automatically adapting the parameters of the weights to the training data is that the objective function is no longer a convex function (Bishop, 2007). This translates in a more lengthy training process, but the model is fast to process the testing data.

Since the high dimensionality of the data does not represent an obstacle in implementing the Neural Network classifier, as it was the case for the GMM, all the HoG features are used. Thus, the considered structure of the network has 2268 input nodes for the body classifier.

\subsection{SVM}

The last HoG based classifiers is Support Vector Machine. The motivation for this choice is the good performance obtained in various classification tasks, particularly in object recognition, where features such as the HoG descriptors are used.

Although at its core the SVM is a 2-class, various techniques and algorithms have been developed for multi-class classification and probability estimates for each of the classes. For our method, we use the variant described in (Wu et al., 2004), which allows multi-class classification with soft assignments (probability estimates), as it can be integrated seamlessly in the probabilistic framework previously described.

\subsection{Velocity}

The velocity direction often represents a cue for the body orientation. However, two factors affect the precision of this cue: the inaccuracy of the estimation of 3D position for the targets and the dependency on the speed of the target. The first disadvantage represents a limitation of the tracker. The second observation

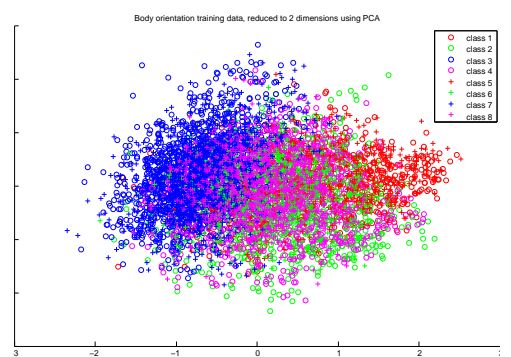

Figure 4: HoG descriptors reduced to 2 dimensions using PCA, where circles and plus signs indicate the opposite directions.

relies on the assumption that a target with a high velocity has a lower chance of changing its orientation than one with a low velocity.

To make better use of both velocity direction and speed, as well as to incorporate this information seamlessly into the previously described framework, we build a pseudo-classifier by defining a Gaussian probability distribution centered around the angle class corresponding to the velocity direction and with a variance inversely proportional to the speed of the target. For a target moving with high speed, the probability of the target facing the movement direction is relatively high, while a near-stationary target will have a near equal probability for all angle classes, as the Gaussian with a high variance will be close to an uniform distribution across all angles.

$l_{\text {velocity }}(\mathbf{x} \mid \alpha)=\log P_{\text {velocity }}(\mathbf{x} \mid \alpha)=\log \mathcal{N}\left(\alpha \mid v_{d}, 1 / v_{m}\right)$

where $v_{d}$ and $v_{m}$ represent the velocity direction and magnitude, respectively. $\mathcal{N}$ denotes the normal distribution.

\subsection{Face Detection}

One inherent limitation of the classifiers based on HoG descriptors is that, given the relatively low resolution of individual targets, the HoG descriptor can only represent the rough outline of the human body. This causes a problem, since usually the appearance of the human body outline is similar for diametrically opposed angles, as suggested in Figure 4. In such cases, a strong cue differentiating the two orientations is the presence of a face.

Face detection can be performed relatively fast, using for example a cascade Local Binary Pattern classifier (Liao et al., 2007). Furthermore, this classifier is able to provide information regarding the type of face detection, i.e. frontal, left-lateral or rightlateral, further aiding the orientation estimation process. 
Given the probabilistic framework described so far, a reasonable approach to model this information is by using an uniform probability distribution over the values of the angle corresponding to the body orientations in which the presence of a face is plausible. Thus, the associated likelihood becomes:

$$
\begin{array}{r}
l_{\text {face }}(\mathbf{x} \mid \alpha)=\log P_{\text {face }}(\mathbf{x} \mid \alpha) \\
P_{\text {face }}(\mathbf{x} \mid \alpha)= \begin{cases}1 / 5 & \text { if } f_{d} \neq 0 \text { and } \alpha \in\{1,5,6,7,8\} \\
0 & \text { if } f_{d} \neq 0 \text { and } \alpha \in\{2,3,4\} \\
1 / 8 & \text { if } f_{d}=0\end{cases}
\end{array}
$$

Note that, the numerical values of the above equation correspond to the values of the uniform distribution. Thus, the first two lines correspond to the situation in which a face is detected $\left(f_{d} \neq 0\right)$ and the probability is uniformly distributed over the 5 angles in which the face can be visible (first line), and all other angles have a zero probability (second line). Lastly, if no face is detected $\left(f_{d}=0\right)$, the probability is evenly distributed among all angles (as the lack of a face detection does not necessarily imply the absence of a face in the image).

\subsection{Temporal Smoothness}

Another cue for the orientation estimation is based on the observation that human targets do not usually change their orientation suddenly from frame to frame, especially considering the fact that frames succeed themselves at least at 1/24 seconds in most video sequences. This can be regarded as a temporal smoothness of the orientation angle.

Thus, to restrict the abrupt changes in estimated orientation angles, we implement a sliding window approach in which the final estimated angle is determined by a majority vote from the angle estimations of the current frame and the past 5 frames (window size was determined empirically). If there is a tie between the angle class estimated for the current frame and another value, the former will be taken.

\section{EXPERIMENTAL RESULTS}

The proposed method for orientation estimation has several hyperparameters which influence the quality of the classification. These hyperparameters are the number of dimensions to which the PCA reduces the HoG descriptors for the GMM, the number of components in each GMM, the number of neurons in the hidden layer of the Neural Network and the kernel type used for the SVM.

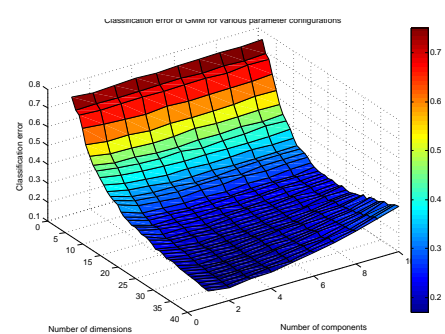

Figure 5: Classification error of the GMM classifier for various parameter configurations (the number of dimensions and the number of components), during validation stage.

To determine suitable values for these hyperparameters, we employ a $k$-fold cross-validation procedure using the available training dataset. Thus, for each parameter configuration of a given classifier, its classification accuracy was computed as an average over the values obtained by training the classifier with a fraction of $(k-1) / k$ of the dataset and estimating the accuracy on the remaining $1 / k$ fraction of the dataset. The results of the cross-validation for each of the classifiers are given in the following paragraphs.

GMM Validation. For determining the hyperparameters of the GMM classifier, namely the number of dimensions to which the PCA reduces the HoG descriptors to and the number of components in each mixture, we employed 4-fold cross validation.

The results of the cross-validation are presented in Figure 5. It can be observed that for relatively low numbers of dimensions, the performance of the classifier is poor, as some information is lost in the dimensionality reduction process, making robust classification difficult. The performance improves significantly after 20 dimensions and it stabilizes between 30 and 40 dimensions, suggesting that the high-dimensional HoG features lie in a lower, 40 dimensional, manifold.

The number of components in each mixture has less impact on the performance, when compared to the number of dimensions. However, the higher error obtained for a single component indicates that the data has a more complex structure than a simple Gaussian distribution, while a high number suggests overfitting, as the performance drops. The best values are obtained for 2-3 components per mixture, these providing the best approximation of the real structure of the data.

Neural Network Validation. The Neural Network classifier has a single parameter, namely the number of neurons in the hidden layer.

The evolution of the classification error is shown in Figure 6. The decreasing evolution of the classification error stabilized after a value approximately 60 


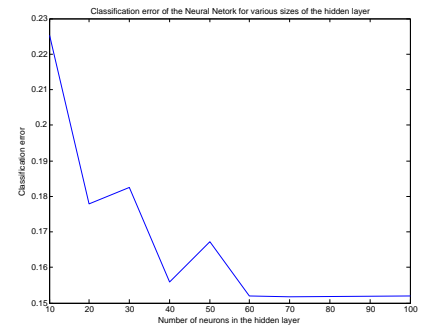

Figure 6: Classification error of the NN classifier for various parameter configurations, during validation stage.

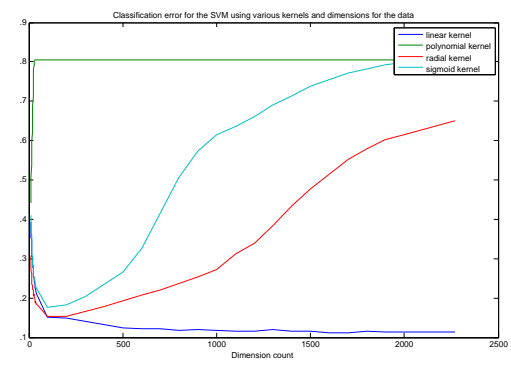

Figure 7: Classification error of the SVM classifier for various parameter configurations, during validation stage.

nodes. Although it is impossible to assess the role of each neuron and thus to provide a solid explanation for the correlation between the number of neurons and performance of the network, one can argue that this size of the hidden layer is influenced by the number of relevant features in the data, similarly to the minimum number of dimensions that yield reasonable good results. Should that be the case, the activation of each neuron is more heavily influenced by one of these implicit relevant features.

SVM Validation. For the SVM classifier, our initial intention was to also employ dimensionality reduction on the features, to obtain faster training times. However, after assessing the performance for various dimensions, as shown in Figure 7, and considering manageable training durations, we decided to use all 2268 HoG dimensions for the SVM classifier.

The plot from Figure 7 shows the evolution of the SVM classification error for various dimensions and using several kernel functions, where we found that the best performed kernel is the linear one.

\subsection{Dataset Description}

During the training of the classifiers we used several datasets, to have a greater variety of appearances. This, in turn, would be beneficial to achieve a better generalization of the training data and a good exploitation of the existing patterns. Some characteris- tics of the datasets used during training are given in Table 1.

For testing the proposed method, we used video sequences from the Collective Activity dataset (Choi et al., 2011), which depict multiple human targets moving unrestricted in an urban environment. The ground truth annotation is available once every 10 frames.

\subsection{Results and Discussion}

The results of the experiments to evaluate the performance of our method are presented in Table 2.

Overall, the performances of the individual classifiers vary to some extent. These variations ensures the capability of a combination of classifiers to yield better results. A certain dependence on the video sequence can also be observed, as all the classifiers obtained better results on Seq 42 than Seq 15. Since these classifiers take into consideration only the visual appearance of the targets, modeled by the HoG descriptors, the only explanation for this behaviour is the fact that the targets from Seq 42 resemble more closely the targets used for the training of the classifier. This visual resemblance can further be explained by a closer similarity of the angle of the camera at which the images were captured, as well as a similarity of the resolution of the images.

The error obtained by combining the response of multiple classifiers proved to be better than the individual responses. Thus, in the case of Seq 42, all the combined responses yielded better results than the individual ones. As expected, the combinations including the more robust classifiers, such as GMM+NN, outperform the ones with the lower performing ones, such as GMM+SVM. In the case of Seq 15, the more pronounced poor result of the SVM classifier has a detrimental impact on the combined responses. Thus, only the GMM+NN combination has a better performance than any of its components, all others being roughly similar or even worse than the individual components.

The second goal of these experiments was to assess the impact of the individual cues considered. The performance of the method when only the velocity is used, proves to be better than the responses of any of the individual or combined HoG-based classifiers, for the considered video sequences Seq 15 and Seq 42, thus highlighting the importance of this additional cue. However, one might expect that for more particular video sequences in which the targets are mostly stationary, the velocity cue would provide less information and thus yield poorer results. The next configuration tested was the combination of the response of 
Table 1: Number of data points per class for the datasets used during training.

\begin{tabular}{|l|c|c|c|c|c|c|c|c|c|c|}
\hline Dataset & Type & C1 & C2 & C3 & C4 & C5 & C6 & C7 & C8 & Total \\
\hline $\begin{array}{l}\text { (M. Andriluka and Schiele., } \\
\text { 2010) }\end{array}$ & Body & 400 & 749 & 644 & 749 & 400 & 622 & 545 & 622 & 4731 \\
\hline (Gernimo et al., 2007) & Body & 129 & 30 & 117 & 78 & 114 & 25 & 141 & 62 & 696 \\
\hline MIT Pedestrian & Body & 0 & 0 & 478 & 0 & 0 & 0 & 446 & 0 & 924 \\
\hline VIPeR & Body & 355 & 90 & 218 & 17 & 6 & 31 & 419 & 126 & 1262 \\
\hline
\end{tabular}

Table 2: Mean and standard deviation of the error for various versions of our method on two video sequences from Collective Activity dataset (Choi et al., 2011).

\begin{tabular}{|l|c|c|}
\hline Method & Seq 15 & Seq 42 \\
\hline GMM & $63.0446 / 28.1862$ & $65.5102 / 31.1502$ \\
\hline NN & $69.7277 / 30.5169$ & $56.3265 / 29.4306$ \\
\hline SVM & $82.2030 / 33.6133$ & $59.6939 / 30.1965$ \\
\hline GMM+NN & $61.7079 / 28.7315$ & $52.6531 / 28.4356$ \\
\hline GMM+SVM & $63.0446 / 28.9955$ & $55.4082 / 29.1968$ \\
\hline NN+SVM & $70.6188 / 31.0409$ & $50.5102 / 27.5921$ \\
\hline GMM+SVM+NN & $66.8317 / 30.2219$ & $54.1837 / 28.8972$ \\
\hline GMM+SVM+NN + Velocity & $48.3416 / 24.7429$ & $36.4286 / 23.2556$ \\
\hline GMM+SVM+NN + Face & $47.6733 / 24.8099$ & $59.0816 / 30.2519$ \\
\hline GMM+SVM+NN + Velocity + Face & $37.4257 / 21.1888$ & $42.5510 / 25.3564$ \\
\hline GMM+SVM+NN + Velocity + Face + Temporal & $38.9851 / 22.0431$ & $23.2653 / 18.9088$ \\
\hline
\end{tabular}

the HoG-based classifiers and the velocity cue. A significant improvement was observed over the response of the HoG-based classifiers, for both videos. However, in the case of Seq 15, where the HoG-based classifiers yielded poor performance, the overall result when taking into account the velocity cue was worse than in the case of using just the velocity. This was not the case for Seq 42, where the performance decreased dramatically, the mean error being lower than either of the constituents' responses.

Next, the face detection cue was assessed, also in combination with the response of the HoG-based classifiers. For Seq 15 the performance improved in a similar fashion to the velocity cue, suggesting a similar informational gain. However, in the case of Seq 42 the performance dropped over one of the HoG-based classifiers, most probably due to the high number of false face detections. When combining the two cues, velocity and face detection, the performance increases in the case of Seq 15, where the two cues taken individually generate similar results, while in the case of Seq 42, the performance is still lower than in the case of using just the velocity cue, due to the poor performance given by the false face detection.

The last element tested was the effect of the temporal smoothing. When combined with the response of the HoG-based classifiers, the performance increased, moderately for Seq 15 and more significantly for Seq 42. The larger improvement in the second case can be explained by a higher number of misclassification, whose influence is reduced. When combined with only the velocity cue, the performance drops slightly for the first video, but increases for the second. This can be explained by a better velocity estimation in Seq 15, in which case the temporal smoothing only delays in response. The increase in the second case is also probably explained by inaccurate estimations of the velocity. Similar trends are followed in the last configuration, involving all classifiers and cues, where the temporal smoothness factor has little influence on the performance from Seq 15 , in which the estimations provided by the classifiers, the velocity and face detections seem to be more reliable. On the other hand, in the case of Seq 42, the performance increase is significant, as the error drops to almost half, due to the fact that misclassification, inexact velocity estimation and false face detections, are smoothed out.

We have compared our method with the state of the art method (Chen and Odobez, 2012), and the results being presented in Table 3. Note that, since the computation time is proportional to the squared number of training and testing data points, only a fraction of the training set was used. For a better comparison, the results of our method using the same reduced training set are also presented. 
Table 3: Evaluation of the performance on a video sequence (Seq 15 from Collective Activity dataset (Choi et al., 2011)) of the original method from (Chen and Odobez, 2012) and our approach.

\begin{tabular}{|l|l|l|l|}
\hline Method & Error & StdDev & $\begin{array}{l}\text { Average pro- } \\
\text { cessing time } \\
\text { per frame } \\
(\mathrm{ms})\end{array}$ \\
\hline $\begin{array}{l}\text { (Chen and } \\
\begin{array}{l}\text { Odobez, } \\
\text { 2012) }\end{array}\end{array}$ & 79.52 & 30.37 & 29948 \\
\hline Our approach & 56.58 & 22.73 & 73 \\
\hline
\end{tabular}

\section{CONCLUSIONS}

We have proposed a novel method for estimating human body orientation from a video based on a committee based approach. One of the benefits of our method is the faster computation time compared to the state of the art method (Chen and Odobez, 2012). Our method also allows for the use of multiple classifiers, their individual responses being combined for a more robust prediction. Another contribution refers to the use of additional cues, such as face detections and temporal smoothness, as well as an improved method on the use of the velocity cue.

\section{ACKNOWLEDGEMENTS}

This research was supported by the FES project COMMIT/.

\section{REFERENCES}

Bishop, C. M. (2007). Pattern recognition and machine learning (information science and statistics).

Chen, C. and Odobez, J. (2012). We are not contortionists: coupled adaptive learning for head and body orientation estimation in surveillance video. In Computer Vision and Pattern Recognition (CVPR), 2012 IEEE Conference on, pages 1544-1551. IEEE.

Choi, W., Pantofaru, C., and Savarese, S. (2012). A general framework for tracking multiple people from a moving camera. IEEE Trans. on Pattern Analysis and Machine Intelligence.

Choi, W., Shahid, K., and Savarese, S. (2011). Collective activity dataset. www.eecs.umich.edu/vision/activitydataset.html.

Dalal, N. and Triggs, B. (2005). Histograms of oriented gradients for human detection. In Computer Vision and Pattern Recognition, 2005. CVPR 2005. IEEE Com- puter Society Conference on, volume 1, pages 886893. IEEE.

Gernimo, D., Sappa, A., Lpez, A., and Ponsa, D. (2007). Adaptive image sampling and windows classification for on-board pedestrian detection. International Conference on Computer Vision Systems.

Hotelling, H. (1933). Analysis of a complex of statistical variables into principal components. Journal of educational psychology, 24(6):417.

Liao, S., Zhu, X., Lei, Z., Zhang, L., and Li, S. Z. (2007). Learning multi-scale block local binary patterns for face recognition. In Advances in Biometrics, pages 828-837. Springer.

Lu, W.-L. and Little, J. J. (2006). Simultaneous tracking and action recognition using the pca-hog descriptor. In Computer and Robot Vision, 2006. The 3rd Canadian Conference on, pages 6-6. IEEE.

M. Andriluka, S. R. and Schiele., B. (2010). Monocular $3 \mathrm{~d}$ pose estimation and tracking by detection. CVPR, www.d2.mpi-inf.mpg.de/node/428.

Tosato, D., Spera, M., Cristani, M., and Murino, V. (2012). Characterizing humans on riemannian manifolds. Pattern Analysis and Machine Intelligence, IEEE Transactions on.

Wang, J. (2011). Geometric structure of high-dimensional data. In Geometric Structure of High-Dimensional Data and Dimensionality Reduction, pages 51-77. Springer.

Wu, T.-F., Lin, C.-J., and Weng, R. C. (2004). Probability estimates for multi-class classification by pairwise coupling. The Journal of Machine Learning Research, 5:975-1005. 